\title{
Pulmonary manifestations of systemic vasculitis in childhood
}

The systemic vasculitides are a heterogenous group of rare conditions with an incompletely understood aetiology. Any of the systemic vasculitides may cause respiratory disease, but some conditions are more likely to affect the pulmonary system, often through pulmonary infarction and diffuse alveolar haemorrhage. These conditions are often difficult to diagnose due to their rarity and significant clinical overlap with common respiratory conditions. Prompt diagnosis and management can significantly reduce morbidity and mortality.

\section{Educational aims}

- To give an overview of the systemic vasculitides of childhood.

- To explore how pulmonary vasculitis manifests in the paediatric population.
Vasculitis is inflammation of blood vessel walls. It can occur secondary to conditions such as infection or connective tissue disease, and as a primary condition in a group of disorders collectively termed the systemic vasculitides. This is a rare group of associated conditions with an estimated prevalence of 23 cases per 100000 children.

It is useful to consider the systemic vasculitides as three entities with separate management strategies: Kawasaki disease and IgA vasculitis (IgAV) make up almost three quarters of paediatric vasculitis cases, and a larger group of rare paediatric vasculitides make up the remainder.

Systemic vasculitides may be difficult to diagnose due to their rarity, a varied presentation depending on which vessels are involved, and significant cross-over of clinical presentation with other more common diseases. All vasculitides have the ability to cause pulmonary injury, but some conditions more commonly affect the lung than others. This review will explore the systemic vasculitides in childhood, focusing on pulmonary injury in these conditions.

\section{Search strategy}

References were identified by searches of MEDLINE (from January 1951 to June 2020), EMBASE (from January 1974 to June 2020), and online Cochrane databases (2020) for the subject headings "systemic
@ERSpublications

The systemic vasculitides are often difficult to diagnose due to their rarity and significant clinical overlap with common respiratory conditions. Prompt diagnosis and management can reduce associated morbidity and mortality. https://bit.ly/36M5tTB
Cite as: Lawton A, Machta J, Semple T, et al. Pulmonary manifestations of systemic vasculitis in childhood. Breathe 2020; 16: 200211. 


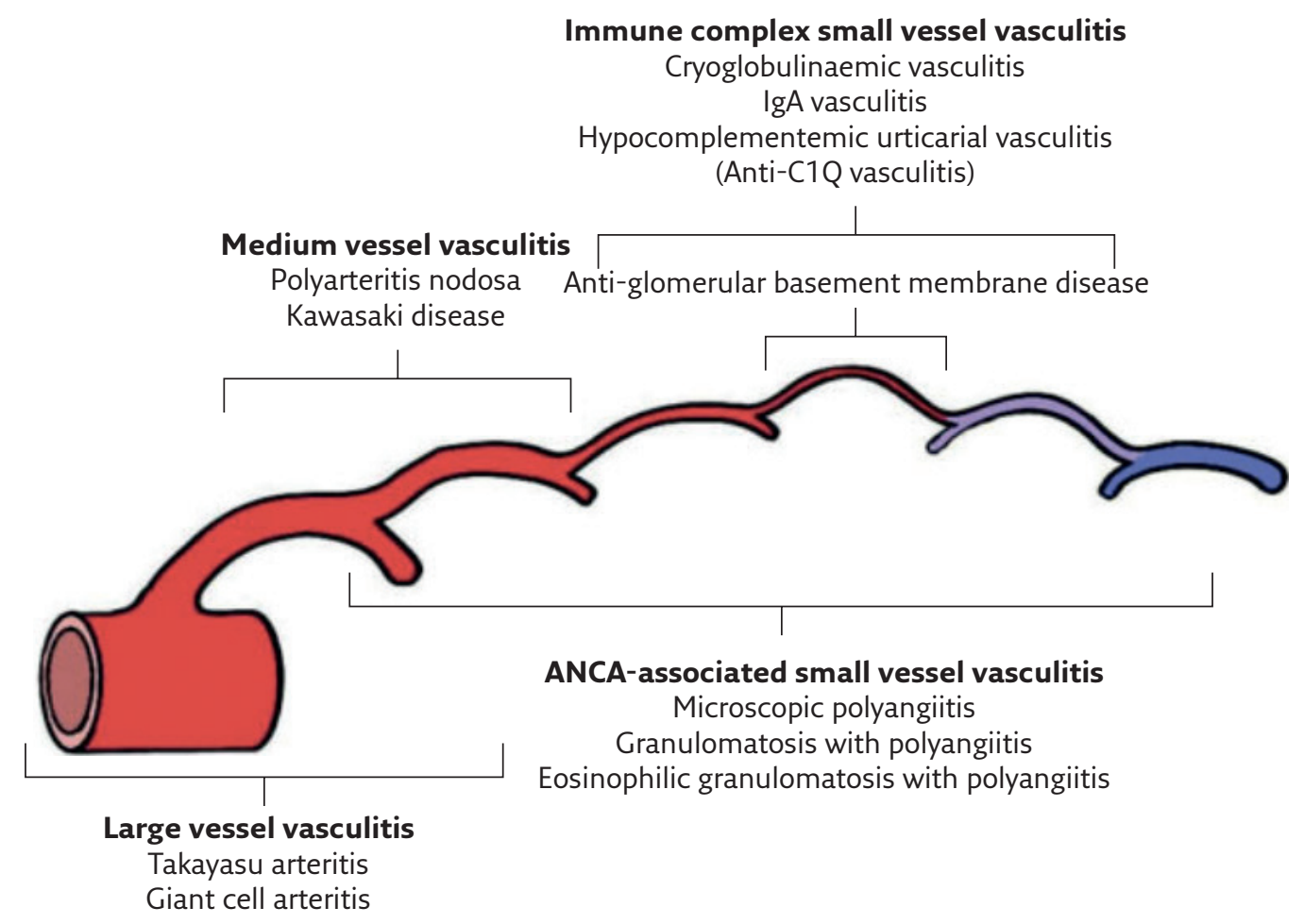

Figure 1 Distribution of vessel involvement in the systemic vasculitides. ANCA: antineutrophil cytoplasmic antibodies. Reproduced from [1], with permission.

vasculitis", "pediatric vasculitis", "paediatric vasculitis" and "pulmonary vasculitis". Only manuscripts published in English were included. Articles were chosen according to their relevance for this review, and their bibliographies were also searched for references. Articles from our personal archives were also included.

\section{Pulmonary vasculature}

To appreciate the consequences of blood vessel damage in pulmonary tissue it is important to review the anatomical peculiarities of the lungs, specifically the presence of a dual circulation: the bronchial and pulmonary circulations.

The pulmonary circulation transports deoxygenated blood from the right ventricle via the pulmonary arteries to the alveoli in order to facilitate gas exchange. This oxygenated blood then returns to the left atrium via the pulmonary veins. Approximately $2 \%$ of this oxygenated blood then passes via the left ventricle and aorta into the bronchial circulation. Bronchial arteries branch from the aorta to supply the bronchi, walls of larger blood vessels, hilar lymph nodes, and visceral pleura with oxygenated blood. While a small percentage of this blood drains back into the systemic circulation via the bronchial veins, most passes into the pulmonary circulation via anastomoses between the two systems.
Inflammation of any of these vessels can occur in vasculitis (figure 1). The location, distribution and severity of the disease in the affected vessels will dictate the clinical consequences. If pulmonary capillaries become affected they may leak and spill erythrocytes into the airspaces, resulting in diffuse alveolar haemorrhage (box 1 and figure 2). In larger pulmonary vessels, infiltration of the vessel wall might cause aneurysm, or narrow and occlude the vessel leading to ischaemia and infarction of the supplied tissue (figure 3).

\section{Pathophysiology of systemic vasculitis}

The aetiology of the systemic vasculitides is not completely understood, but is thought to be multifactorial in nature, including abnormal immune activation in individuals with a genetic predisposition to developing vasculitis. Immune activation explains why the systemic vasculitides may be confused at first presentation with infection, because they may present with constitutional symptoms such as fever, malaise, and weight loss.

There are several abnormal immune responses that can ultimately result in vasculitis. First, immune complexes (antibodies bound to an antigen) may be deposited in blood vessel walls, triggering inflammation. Secondly, abnormal 
Box 1 Diffuse alveolar haemorrhage (DAH) (adapted from [2])

\section{Presenting features of DAH}

DAH may be asymptomatic, or may present with one or more of:

- Dyspnoea

- Haemoptysis (seen in one third of cases)

- Acute fall in haematocrit

\section{Radiological findings}

- Chest radiography may be normal or show pulmonary infiltrates.

- In the acute phase, computed tomography (CT) findings are nonspecific, but may include ground-glass opacification or consolidation, generally sparing the lung peripheries, and enlarged hilar lymph nodes.

- Progression to the formation of interlobular septal thickening and emphysema suggests the development of haemosiderosis.

\section{Bronchoscopy findings}

- Bronchoscopy is helpful to exclude other causes of haemoptysis.

- Bronchoalveolar lavage with saline has several functions in the diagnosis of DAH:

1) Allows retrieval of specimens to culture for investigation of possible infectious causes.

2) Instilling and removing saline from the airways damages the already inflamed capillaries and causes them to bleed. The fluid instilled in subsequent aliquots becomes increasingly blood-stained as fresh bleeding occurs. This is diagnostic of DAH.

\section{Pathological findings}

- Microscopy demonstrates haemosiderin-laden macrophages following DAH, as erythrocytes are phagocytosed by macrophages and haemoglobin is broken down into haemosiderin.

\section{Management}

- Treatment of the systemic vasculitis will cause resolution of symptoms, but this takes time to occur following initiation of immunosuppressants.

- Control of symptoms and management of respiratory failure may be required in the meantime. This includes consideration of mechanical ventilation and intensive care unit (ICU) care.

- Extracorporeal membrane oxygenation has been successfully used in some cases as a bridging treatment for respiratory failure until the induction of disease remission is achieved.

leukocyte functioning allows leukocytes to directly attack blood vessel walls. Finally, autoantibodies can be generated which activate neutrophils to cause endothelial injury [3].

Biopsy is often required to confirm a diagnosis of vasculitis. When examined histologically, findings include blood vessel inflammation which may progress to thrombosis and necrosis of supplied tissue. Necrotic pulmonary lesions may be appreciated radiologically as discrete nodules, which may cavitate. Granulomas (organised collections of inflammatory cells, mostly macrophages) may also be seen in some conditions.

\section{Classification of vasculitis}

There are no agreed diagnostic criteria for many of the systemic vasculitides, but classification criteria have been developed in order to define consistent patient groups for research purposes. Classification criteria cannot be used to make a diagnosis in individual patients, who may fail to meet these criteria but still have vasculitis. For many conditions the definitive diagnosis of vasculitis requires histopathological confirmation from biopsy of the affected sites, a lung biopsy is seldom required due to the frequent availability of safer biopsy targets such as skin lesions.

In 2005, the European League Against Rheumatism (EULAR) and the Paediatric Rheumatology European Society (PRES) established a framework to classify childhood vasculitides [4]. The EULAR/PRES classification was based on a modification of the Chapel Hill criteria used for adult vasculitides, which chose predominantly affected vessel size as the main discriminating feature. The categorisation of paediatric vasculitides has taken a similar approach, with attention also paid to the presence or absence of granulomatous disease (table 1). The EULAR/PRES classification includes a category for vasculitis secondary to other causes: including medication, infection and connective tissue disorders. In 2008, EULAR and PRES together with the Paediatric Rheumatology International Trials Organisation (PRINTO) published a formal statistical validation of the EULAR/PRES classification, and defined classification criteria for several types of vasculitis, these are referred to as the EULAR/PRINTO/PRES criteria [5]. 

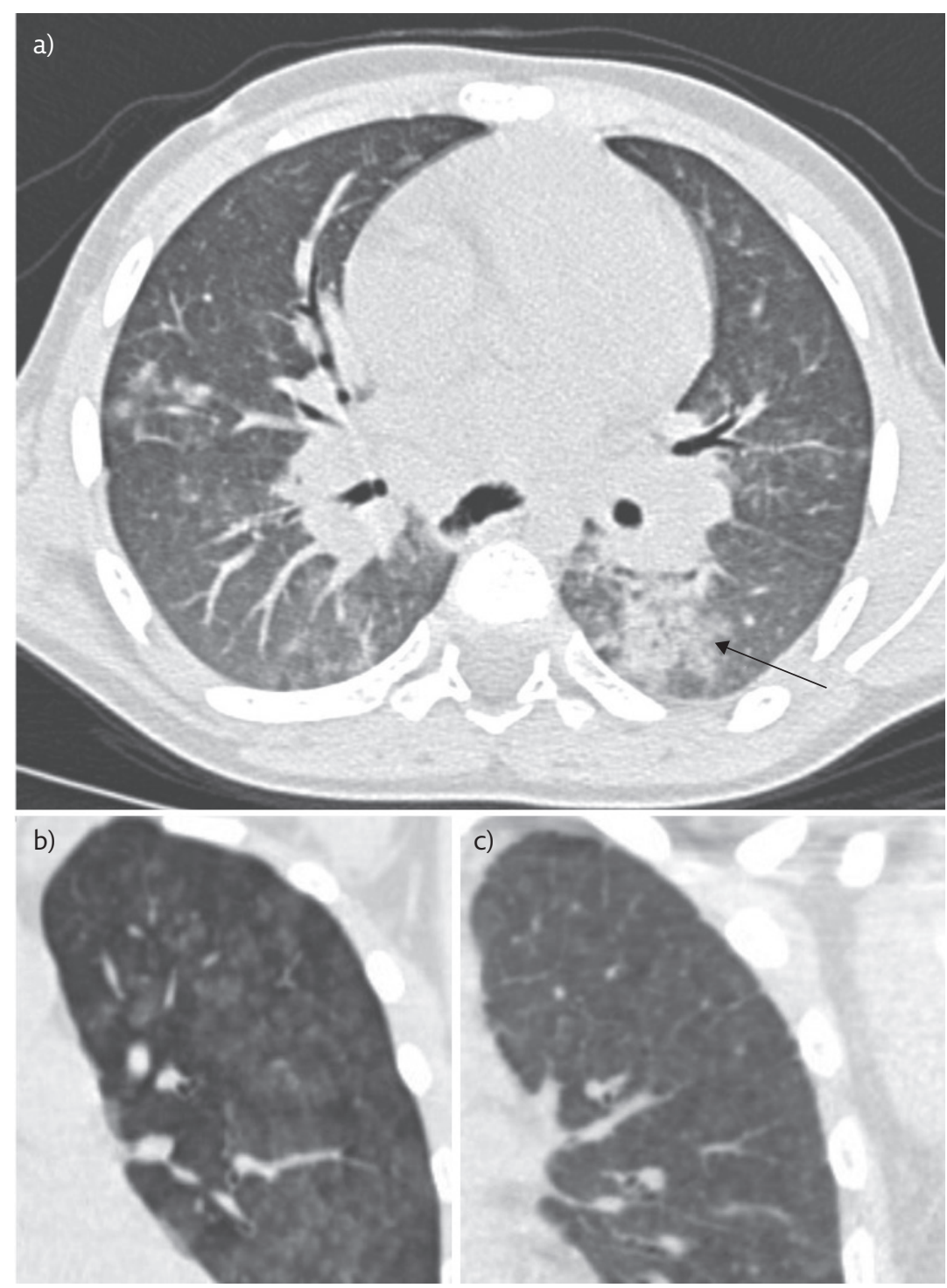

Figure 2 Pulmonary haemorrhage. a) A 10-year-old girl with nodular ground-glass opacification (arrow) due to pulmonary haemorrhage b) A 9-year-old girl with idiopathic pulmonary haemorrhage, c) follow-up of this child a year later demonstrates more diffuse ground-glass opacification with interlobular septal thickening, in keeping with the development of chronic pulmonary haemosiderosis.
The management of IgAV and Kawasaki disease are described in later sections of this review, and the management of the rare paediatric vasculitides are collectively explored here to avoid repetition.

Treatment of the rare paediatric vasculitides focuses on induction and maintenance of disease remission using glucocorticoids and other immunosuppressive agents (box 2). Supportive care includes antiplatelet agents that may prevent thrombosis, as well as prophylaxis for the consequences of long-term steroid use; prophylactic antibiotic therapy against Pneumocystis jirovecii; proton pump inhibitors for gastric protection; and osteoporosis prophylaxis.

\section{Large vessel vasculitis: childhood-onset Takayasu arteritis}

c-TA is a chronic, granulomatous, large-vessel vasculitis that most commonly involves the aorta, its major branches and the pulmonary arteries [9]. It has been known to cause stenosis, occlusion or aneurysm of any of these vessels.

Stenosis of the large arteries leads to arterial hypertension in $80 \%$ of children, which is the most common clinical finding [10]. Occlusion of larger vessels can result in a loss of peripheral pulses, particularly in the upper limbs, and for this reason it has been known as the "pulseless disease". Occlusion of pulmonary arteries may rarely lead to pulmonary hypertension which can be lifethreatening, as can the development and rupture of pulmonary artery aneurysms. Angiographic abnormalities of large vessels are essential to the classification of c-TA (box 3), either using CT angiography or magnetic resonance imaging (MRI). $\mathrm{MRI}$ is the preferred modality in children due to the avoidance of radiation exposure.

Wheeze, pleural effusions and respiratory failure have all been described in C-TA [11]. Pulmonary involvement occurred in a third of patients in one Chinese cohort, with angiographic evidence of pulmonary artery involvement in $12 \%$ of children [12].

Arterial hypertension must be controlled to reduce the risk of end organ damage. Screening for end organ damage should be undertaken, including formal ophthalmological assessment. End organ injury or drug-resistant hypertension should prompt consideration of surgical revascularisation procedures.

c-TA should be considered as a differential diagnosis whenever a child presents with pulmonary artery aneurysms, or with multiple areas of pulmonary infarction but without obvious risk factors for thromboembolic disease.

\section{Medium vessel vasculitis}

The commonest of these conditions is Kawasaki disease which, by some estimates, accounts for 
almost half of all paediatric vasculitis. c-PAN is significantly rarer, but is notable for its ability to cause bronchial artery aneurysms that might be identified following investigation for haemoptysis, or seen incidentally in asymptomatic patients [13].

\section{Kawasaki disease}

Kawasaki disease is notorious for its most significant complication: coronary artery aneurysm. It is probably caused by an abnormal immune response to an unidentified infection in genetically predisposed children [14]. The diagnostic criteria for Kawasaki disease require 5 days of fever with at least four of the following features: cervical lymphadenopathy, bilateral non-purulent conjunctivitis, diffuse rash, erythema or oedema of the extremities, and oral changes including erythema of the oral mucosa, cracked lips or strawberry tongue [15].

Respiratory presentations (involving cough and respiratory distress) are rare, presenting in fewer than $2 \%$ of patients in one large Indian cohort [16]. All 11 children with respiratory presentations in this cohort had radiographic evidence of consolidation. Half of these children also had pleural effusions, and there were also three cases of empyema and two cases of pneumothorax.

The management of Kawasaki disease includes a combination of aspirin and intravenous immunoglobulin (IVIG) to reduce the incidence of coronary artery aneurysms, and repeat echocardiography to monitor the coronary arteries. Although primary pulmonary disease in Kawasaki disease is rare, IVIG is derived from donated human blood and like all blood products may cause transfusion-related acute lung injury. This is defined as the onset of new respiratory distress occurring within $6 \mathrm{~h}$ of a transfusion, and is associated with non-cardiogenic pulmonary oedema [17]. It has been previously described following IVIG treatment for Kawasaki disease [18].

\section{Small vessel vasculitis}

The small vessel vasculitides include the relatively common IgAV and a group of rarer conditions collectively termed the ANCA-associated vasculitides (AAVs), named due to their association with antineutrophil cytoplasmic antibodies (ANCA). ANCA are antibodies against a number of self-antigens, most notably proteinase- 3 and myeloperoxidase. They are probably a factor in the pathogenesis of these conditions as they appear strongly immunogenic, and their disappearance from blood is associated with a reduced risk of relapse. The AAVs include GPA, EGPA and MPA.

\section{Immunoglobulin A vasculitis}

IgAV was previously known as Henoch-Schönlein Purpura, but was renamed in 2012 when the
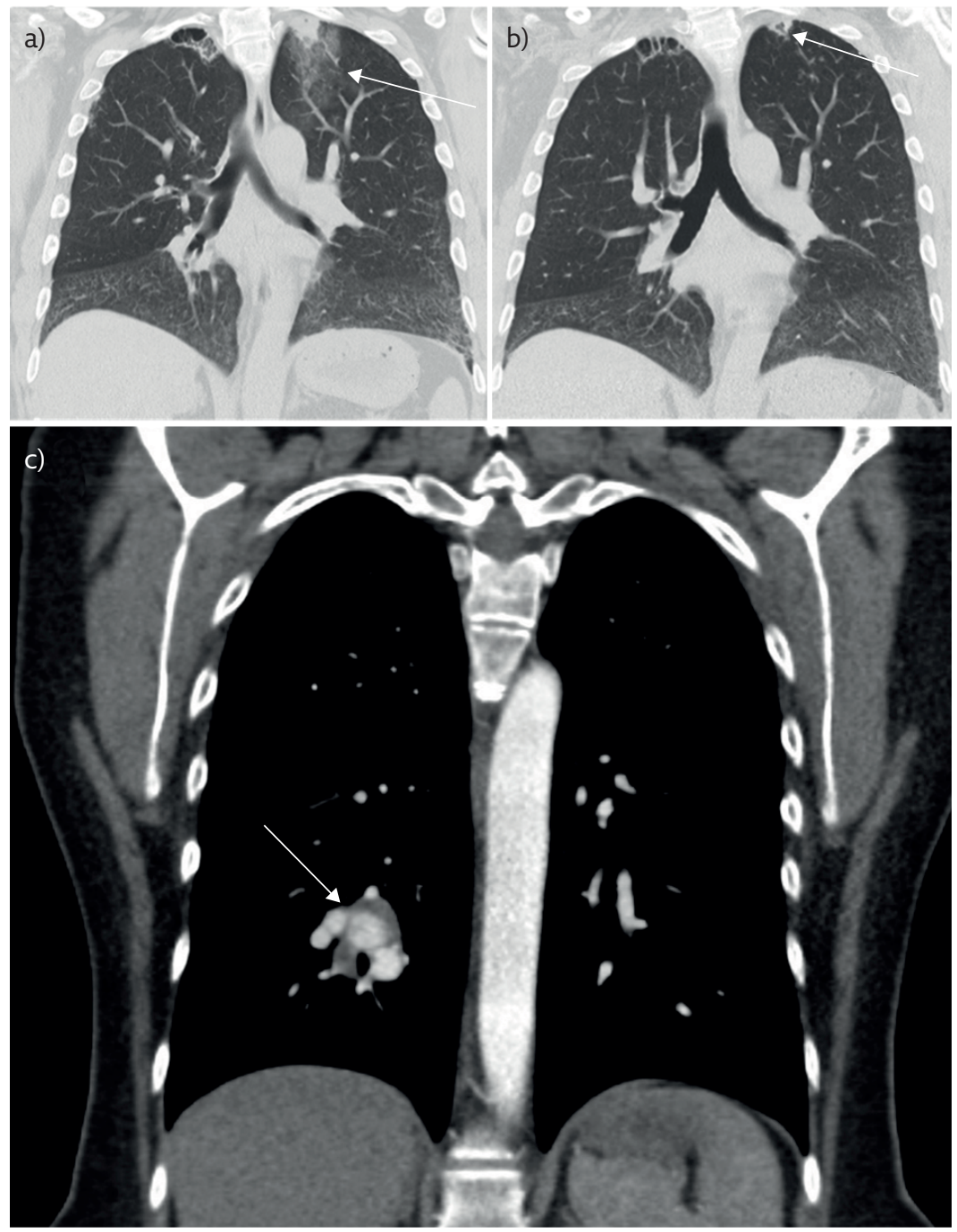

Figure 3 Infarcts and aneurysms secondary to vasculitis. a) Wedge-shaped area of consolidation (arrow) at the left apex with a tiny central cavity and surrounding ground-glass opacification. b) Follow-up CT shows the apical focus has decreased in size, but with larger central cavitation (arrow). This is the expected imaging progression of a pulmonary infarct. c) A different patient with a right lower lobe pulmonary artery aneurysm (arrow) due to Beçhets disease.

nomenclature of the vasculitides was revisited [1]. It is usually preceded by a viral trigger, and many viral species are implicated as potentially causative [19].

EULAR/PRINTO/PRES classification criteria exist for IgAV, and require purpura or petechiae with a predominantly lower limb distribution. There must also be at least one of the following: histological findings supportive of vasculitis, abdominal pain, joint pain, or renal involvement.

It is extremely rare that the respiratory tract is affected in IgAV, although case reports of children with pulmonary haemorrhage exist [20]. In this case, diffuse pulmonary infiltrates were seen on chest radiography, frank blood was seen on endotracheal intubation suggesting pulmonary haemorrhage, and the child required mechanical ventilation. Despite children with IgAV rarely experiencing respiratory symptoms, diffusing capacity of the lungs for carbon monoxide has been found to be consistently low 
Table 1 Classification of childhood vasculitis

\section{Predominantly large vessel vasculitis}

Takayasu arteritis

\section{Predominantly medium sized vessel vasculitis}

Childhood polyarteritis nodosa

Cutaneous polyarteritis

Kawasaki disease

\section{Predominantly small vessel vasculitis}

Granulomatous

Granulomatosis with polyangiitis (GPA)

Eosinophilic granulomatosis with polyangiitis (EGPA)

Non-granulomatous

Microscopic polyangiitis (MPA)

Immunoglobulin A vasculitis (IgAV)

Isolated cutaneous leukocytoclastic vasculitis

Hypocomplementemic urticarial vasculitis (HUV)

\section{Other vasculitis}

Behçet's disease

Vasculitis secondary to infection (including hepatitis B-associated polyarteritis nodosa), malignancies, and drugs, including hypersensitivity vasculitis

Vasculitis associated with connective tissue diseases

Isolated vasculitis of the central nervous system

Cogan syndrome

Unclassified

Reproduced from [4], with permission.
The most common features in children are renal involvement combined with both upper and lower respiratory tract involvement. Constitutional symptoms of weight loss, fever, malaise are seen in around $80 \%$ of patients [22]. The spectrum of pulmonary involvement ranges from asymptomatic radiological abnormalities to DAH (figure 4). Clinically, this may manifest with any of cough, dyspnoea, hypoxaemia, anaemia or haemoptysis. Nodular lung lesions are the commonest chest radiography finding in GPA. The nodules consist of necrotising, granulomatous inflammation, which may cavitate [23]. CT of the thorax may demonstrate the "halo sign": a pulmonary nodule surrounded by a small area of ground-glass opacification representing adjacent haemorrhage [24].

The AAVs have a high morbidity. In one cohort study, half of the children required ICU admission as part of their GPA management, with one third of these admissions for respiratory failure. Of the 28 children included in this cohort, five required mechanical ventilation for respiratory failure [25].

\section{Eosinophilic granulomatosis with polyangiitis}

EGPA (previously known as Churg-Strauss syndrome) is a rare disease with almost universal pulmonary involvement. The largest paediatric cohort study consists of 14 cases from a French dataset of rare respiratory diseases [26].

EGPA is considered to progress through three phases. First, the allergic phase involving asthma with or without sinusitis. Secondly, the eosinophilic stage where high numbers of eosinophils (which are considered to have a role in the pathogenesis of the condition) are seen in both tissue biopsy and on serological testing. Finally, the vasculitic phase with small vessel vasculitis with possible multiorgan involvement.

In the French cohort study, $86 \%$ of patients exhibited non-fixed pulmonary infiltrates on chest radiography. Pulmonary nodules were less frequently seen, and only one of the 14 patients developed pleural effusion. The asthma associated with EGPA in this cohort was relatively severe. Of those with asthma, 92\% were GINA (Global Initiative for Asthma) step 4-5 at diagnosis, meaning they required more than a long-acting beta agonist and a low-dose inhaled corticosteroid to control their symptoms.

\section{Microscopic polyangiitis}

This is a predominantly small vessel vasculitis with frequent renal and pulmonary involvement. It rarely involves the upper respiratory tract and granulomas are not seen on biopsy. No paediatric classification criteria exist for MPA, but renal disease is the most prominent feature and is often severe. Respiratory disease is present in almost half of patients and 
Box 2 Treatment approach in the rare paediatric vasculitides (adapted from [8])

\section{Induction}

- Consider methylprednisolone i.v. 10-30 $\mathrm{mg} \cdot \mathrm{kg}^{-1}$ (maximum of $1 \mathrm{~g}$ per day) for 3 consecutive days, followed by prednisolone $1-2 \mathrm{mg} \cdot \mathrm{kg}^{-1}$ per day (maximum of $60 \mathrm{mg}$ per day), which then tapers over 6 months.

- Cyclophosphamide 500-1000 $\mathrm{mg} \cdot \mathrm{m}^{-2}$ i.v. (maximum of $1.2 \mathrm{~g}$ ) every 3-4 weeks for 3-6 months.

- Aspirin 2-5 mg $\mathrm{kg}^{-1}$ once a day (or dipyridamole $2.5 \mathrm{mg} \cdot \mathrm{kg}^{-1}$ twice a day if aspirin is contraindicated).

- Rituximab or mycophenolate mofetil (MMF) can be used as induction agents in some cases.

\section{Maintenance}

- Minimum duration of 1-3 years.

- Azathioprine (2-3 $\mathrm{mg} \mathrm{kg}^{-1}$ p.o. once a day) or MMF as first line agents.

- Prednisolone $0.1-0.2 \mathrm{mg} \cdot \mathrm{kg}^{-1}$ daily.

- Aspirin 2-5 mg $\mathrm{kg}^{-1}$ once a day (or dipyridamole $2.5 \mathrm{mg} \cdot \mathrm{kg}^{-1}$ twice a day if aspirin is contraindicated).

\section{Stopping treatment}

- If no disease activity is present for a year after stopping prednisolone, consider a slow withdrawal of other drugs over 6 months.

\section{Relapse}

- For minor relapses: increase the dose of prednisolone and consider increasing the dose of other disease modifying anti-rheumatic drugs (DMARDs).

- For major relapses (new onset or recurrence of potentially organ/life threatening disease):

1) Change current DMARD to cyclophosphamide, rituximab or MMF, depending on what induction drug was used. In some cases biological agents (IVIG, tocilizumab, anti-tumour necrosis factor agents) may be tried.

2) Methylprednisolone $10-30 \mathrm{mg} \cdot \mathrm{kg}^{-1}$ (maximum of $1 \mathrm{~g}$ per day), for 3 consecutive days.

3) Increase prednisolone to $1 \mathrm{mg} \cdot \mathrm{kg}^{-1}$ per day, then taper.

4) Consider plasma exchange.

5) Return to a different maintenance agent than previously tried once remission achieved.

\section{Box 3 Classification criteria for specific systemic vasculitides (information from [4])}

\section{c-TA}

1) Specific angiographic abnormalities (including aneurysm, dilatation, narrowing, occlusion and thickening of the arterial wall) of the aorta or its main branches and pulmonary arteries; plus any one of:

2) Pulse deficit or claudication

3) Four limbs blood pressure discrepancy

4) Bruits

5) Hypertension

6) Elevated C-reactive protein or erythrocyte sedimentation rate

\section{IgAV}

1) Purpura or petechiae with lower limb predominance,and at least one of:

2) Abdominal pain

3) Histopathology demonstrating leukocytoclastic vasculitis with IgA deposition

4) Arthritis or arthralgia

5) Renal involvement

\section{GPA}

At least three of:

1) Histopathology (including granulomatous inflammation)

2) Upper airway involvement (including saddle nose deformity, chronic sinusitis)

3) Laryngo-tracheo-bronchial stenoses

4) Pulmonary involvement (radiological evidence of nodules, cavities or fixed infiltrates)

5) ANCA positivity

6) Renal involvement 

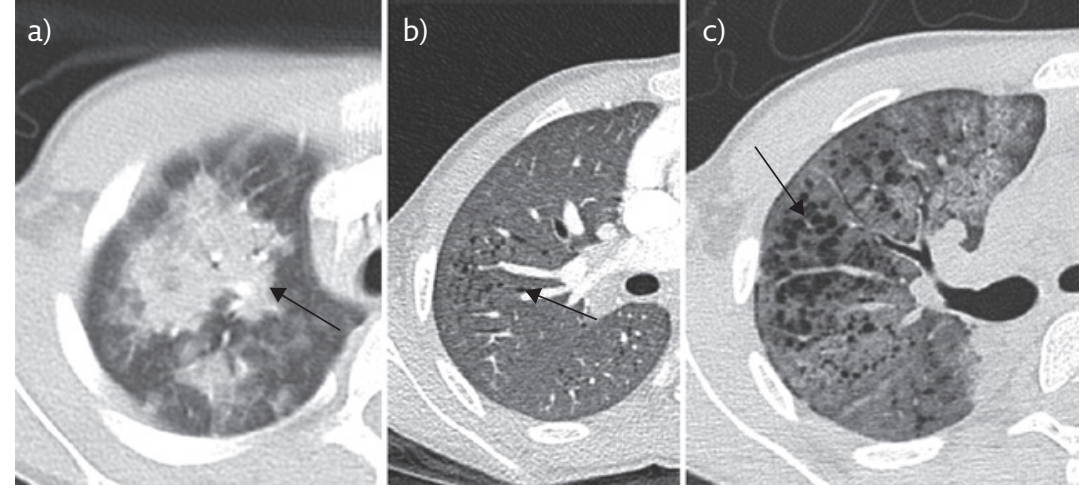

Figure 4 A 10-year-old boy, ANCA positive, presenting with acute haemoptysis and renal failure. a) Peribronchial consolidation (arrow) at the time of acute presentation with haemoptysis. b) 2 years later, progressive ground-glass opacification (arrow) with formation of cysts/emphysema. c) 6 years later, with the development of secondary haemosiderosis (arrow).

ranges from asymptomatic pulmonary small vessel disease to severe cough, haemoptysis and dyspnoea leading to respiratory failure [27].

\section{Other causes of systemic vasculitis}

The 2005 EULAR/PRES classification system for paediatric vasculitides included an "other vasculitides" group for those conditions that either did not fit one of the other categories, fitted in more

Table 2 Drugs known to have caused pulmonary vasculitis

All-trans retinoic acid (ATRA)

Allopurinol

Benzylthiouracil

Carbimazole

Etanercept

Fluoxetine

Golimumab

Hydralazine

Infliximab

Interferon alpha/beta

Levamisole-adulterated/tainted cocaine

mTOR (mammalian target of rapamycin) inhibitors e.g. sirolimus

Methimazole

Nilotinib

Nitrofurantoin

Penicillins

Phenytoin (diphenylhydantoin)

Pristane

Propylthiouracil (PTU)

Information from [28]. than one other category, or had a known aetiology such as infection or drug-induced vasculitis (table 2).

A thorough exploration of other causes of childhood vasculitis is beyond the scope of this review, but particular mention is given to the following conditions that have significant relevance.

\section{Behçet's disease}

Behçet's disease is a systemic inflammatory disease that is rare in the paediatric population. It involves a vasculitis that can affect both arteries and veins of any size. Lung involvement is rare, but can be associated with significant mortality. Pulmonary arteritis is one of the leading causes of death in Behçet's disease [29].

\section{Systemic lupus erythematosus}

Systemic lupus erythematosus is a chronic autoimmune inflammatory disease without a well understood aetiology. Children with systemic lupus erythematosus often have a more severe form of the disease than adults, and alveolar haemorrhage is more common and more severe, with a mortality approaching $70 \%$ in some studies [30].

\section{Hypocomplementemic urticarial vasculitis}

Obstructive lung diseases such as asthma and COPD are associated with this condition, with half of adult patients having COPD. Features include low serum complement and urticarial symptoms lasting longer than 6 months, with vasculitic findings on skin biopsy. Spirometry findings consistent with obstructive lung disease have been reported in the case of a teenager who later developed exertional dyspnoea and emphysematous changes on CT of the thorax [31].

\section{Infection}

Pseudomonas and Legionella infections may cause a necrotising pulmonary vasculitis if a pneumonia becomes complicated by direct infection of the pulmonary vessels [32]. Entry of pathogens into blood vessel walls may cause inflammation and immune-complex deposition, or generation of autoantibodies that trigger vasculitis. Other viruses, bacteria and fungi have been implicated, many of which also cause respiratory disease such as Mycobacterium tuberculosis, Burkholderia cepacia and Streptococcus pneumoniae.

\section{Primary genetic causes of pulmonary vasculitis}

Although rare, the severity of the resulting disease warrants the discussion of two recently described 
genetic vasculitic conditions presenting in infancy or early childhood.

TMEM173 encodes the stimulator of interferon genes (STING), and mutations can result in persistent activation of the STING-interferon pathway. First described in six patients in 2014, this auto-activation results in STING-associated vasculopathy with onset in infancy (SAVI) [33]. All six patients were symptomatic within 8 weeks of birth, presenting with tachypnoea, a telangiectatic, pustular or blistering rash on the cheeks, nose, fingers, toes or soles of the feet and elevated inflammatory markers. Five of these six patients had an interstitial lung disease with lymphocytic infiltrates, fibrosis and emphysema on biopsy. Importantly, as this autoinflammation is secondary to interferon-B pathway activation, treatment via Janus kinase inhibitors may present a viable therapeutic strategy.

In 2015, a similar autosomal dominant autoimmune syndrome was described, associated with variations in the Coatomer subunit alpha (COPA) gene [34]. Of 27 members of five families with mutations in the COPA gene, all had pulmonary disease (pulmonary haemorrhage, interstitial lung disease or both) with an average age of 3.5 years at presentation and $76 \%$ presenting before the age of 5 years.

\section{Summary}

The systemic vasculitides are a heterogenous group of rare conditions that are classified in children according to the size of the predominantly affected blood vessels. Kawasaki disease and IgAV are significantly more common than the rare paediatric vasculitides.

Pulmonary involvement involves a spectrum of clinical findings from asymptomatic patients with radiological or physiological evidence of lung disease, through to cough, dyspnoea, and haemoptysis resulting in respiratory failure, mechanical ventilation and death.

Diagnosis remains challenging due to the rarity of these conditions in comparison to more common pathologies with similar clinical findings. European consensus-based recommendations exist for the diagnosis and treatment of paediatric vasculitides, although these are largely based on expert opinion due to lack of clinical research in this area. Treatment of the rare paediatric vasculitides generally includes immunosuppression with glucocorticoids and other immune-modulating agents. Clinicians should remain alert to the possibility of a systemic vasculitis when faced with a history of unexplained pulmonary disease, particularly when associated with systemic inflammation. When onset is at a very young age (particularly infantile onset) or there is a significant family history of autoimmune disease, consideration should be given to a primary

\section{Self-evaluation questions}

1. A 15-year-old male is seen in clinic with fatigue, myalgia, purpuric rash and fever. He has a background of difficult-to treat asthma for which he is treated with salbutamol as a reliever, and high-dose inhaled beclomethasone as a preventer. He also has multiple food allergies and chronic sinusitis. He undergoes tissue biopsy of the skin to aid with diagnosis. Given the likely underlying diagnosis, what does the biopsy show?

a) Eosinophilia, granuloma formation

b) Eosinophilia, no granuloma formation

c) Thrombosis, necrosis, granuloma

d) Small vessel inflammatory changes

e) No specific abnormal findings

2. A 4-year-old female presents with a 5-day history of fever, cracked lips, a rash of the extremities, cervical lymphadenopathy and irritability. What is the likely diagnosis and management?

a) Childhood-onset Takayasu arteritis, intravenous corticosteroids

b) Kawasaki disease, intravenous immunoglobulin and high-dose Aspirin

c) Kawasaki disease, intravenous corticosteroids

d) IgA Vasculitis, conservative management

e) IgA Vasculitis, intravenous corticosteroids

3. Which of the following statements concerning paediatric vasculitides are true?

a) Childhood-onset Takayasu Arteritis is a small-vessel predominant vasculitis with no reported pulmonary involvement

b) Eosinophilic Granulomatosis with Polyangiitis is a common paediatric vasculitis in which respiratory complications are rare

c) Childhood-onset Takayasu arteritis is a large-vessel predominant vasculitis which may cause pulmonary artery aneurysms

d) Granulomatosis with polyangiitis is an ANCA-negative vasculitis

e) Eosinophilic granulomatosis with polyangiitis is a rare paediatric vasculitis in which respiratory complications are common

4. An 8-year-old male presents to the emergency department with a purpuric rash on the lower limbs and buttocks, abdominal pain, and bilateral arthralgia affecting the knees. Which of the following statements is most true of the likely diagnosis?

a) Granulomatosis with polyangiitis, pulmonary involvement is common

b) Granulomatosis with polyangiitis, pulmonary involvement is rare

c) Microscopic polyangiitis, pulmonary involvement is rare

d) IgA vasculitis, respiratory symptoms are common

e) IgA vasculitis, respiratory symptoms are rare

5. Which of the following is not a proposed immunopathogenic mechanism for the development of vasculitis?

a) Immune complex deposition in blood vessel walls

b) Pathological leukocyte function

c) Genetic mutation preventing synthesis of interferon-B

d) Autoantibodies causing neutrophil-mediated epithelial injury

genetic cause (including the recently described SAVI and COPA). As more is known of the aetiology of autoimmune disease in general, more diseasespecific targeted therapies are likely to emerge. 


\section{Key points}

- The systemic vasculitides are rare and their clinical presentation shares significant overlap with common respiratory conditions, making timely diagnosis challenging.

- Any of the vasculitides can affect the lungs, but some conditions are more likely to do so.

- Pulmonary involvement exists on a spectrum from asymptomatic patients with radiological or physiological evidence of lung disease, through to pulmonary haemorrhage and respiratory failure resulting in death.

- $\quad$ Systemic vasculitis should be considered as a differential diagnosis in unexplained pulmonary disease, especially if associated with systemic inflammation.

\section{Affiliations}

\section{Adam Lawton' ${ }^{1}$ Joseph Machta ${ }^{2}$, Thomas Semple ${ }^{3}$, Atul Gupta 4,5}

${ }^{1}$ Dept of Paediatrics, Royal London Hospital, London, UK. ${ }^{2}$ Royal Free London NHS Foundation Trust, Dept of Paediatrics, London, UK. ${ }^{3}$ Royal Brompton Hospital, Dept of Radiology, London, UK. ${ }^{4}$ Dept of Paediatric Respiratory Medicine, King's College Hospital NHS Foundation Trust, London, UK. Institute for Women's and Children's Health, King's College London, London, UK.

\section{Conflict of interest}

None declared.

\section{Suggested answers}
1. a.
2. b.
3. $c$ and e.
4. e.
5. c.

\section{References}

1. Jennette JC, Falk RJ, Bacon PA, et al. 2012 Revised International Chapel Hill consensus conference nomenclature of vasculitides. Arthritis Rheum 2013; 65: 1-11.

2. Nasser M, Cottin V. Alveolar hemorrhage in vasculitis (primary and secondary). Semin Respir Crit Care Med 2018; 39: 482-493.

3. Brown KK, Frankel SK, Cool CD. Pulmonary Vasculitis. In: Broaddus VC, Mason RJ, Ernst JD, et al., eds. Murray and Nadel's Textbook of Respiratory Medicine. 6th Edn. Philadelphia, Elsevier, 2016; pp. 1068-1069.

4. Ozen S, Ruperto N, Dillon MJ, et al. EULAR/PReS endorsed consensus criteria for the classification of childhood vasculitides. Ann Rheum Dis 2006; 65: 936-941.

5. Ozen S, Pistorio A, Iusan SM, et al. EULAR/PRINTO/PRES criteria for Henoch-Schönlein purpura, childhood polyarteritis nodosa, childhood Wegener granulomatosis and childhood Takayasu arteritis: Ankara 2008. Part II: Final classification criteria. Ann Rheum Dis 2010; 69: 798-806.

6. De Graeff N, Groot N, Ozen S, et al. European consensusbased recommendations for the diagnosis and treatment of Kawasaki disease-the SHARE initiative. Rheumatology (Oxford) 2009; 58: 672-682.

7. Ozen S, Marks SD, Brogan P, et al. European consensusbased recommendations for diagnosis and treatment of immunoglobulin A vasculitis-the SHARE initiative. Rheumatology (Oxford) 2019; 58: 1607-1616.

8. De Graeff N, Groot N, Brogan P, et al. European consensusbased recommendations for the diagnosis and treatment of rare paediatric vasculitides-the SHARE initiative. Rheumatology (Oxford) 2019; 58: 656-671.
9. Mathew AJ, Goel R, Kumar S, et al. Childhood-onset Takayasu arteritis: An update. Int J Rheum Dis 2016; 19 : 116-126.

10. Di Santo M, Stelmaszewski EV, Villa A. Takayasu arteritis in paediatrics. Cardiol Young 2018; 28: 354-361.

11. Eleftheriou D, Varnier G, Dolezalova P, et al. Takayasu arteritis in childhood: Retrospective experience from a tertiary referral centre in the United Kingdom. Arthritis Res Ther 2015; 17: 36.

12. Fan L, Zhang H, Cai J, et al. Clinical course and prognostic factors of childhood Takayasu's arteritis: Over 15-year comprehensive analysis of 101 patients. Arthritis Res Ther 2019; 21: 1-12.

13. Adams TN, Zhang D, Batra K, et al. Pulmonary manifestations of large, medium, and variable vessel vasculitis. Respir Med 2018; 145: 182-191.

14. Dietz SM, van Stijn D, Burgner D, et al. Dissecting Kawasaki disease: a state-of-the-art review. Eur J Pediatr 2017; 176 : 995-1009.

15. McCrindle BW, Rowley AH, Newburger JW, et al. Diagnosis, treatment, and long-term management of Kawasaki disease: A scientific statement for health professionals from the American Heart Association. Circulation 2017; 135: e927-e999.

16. Singh S, Gupta A, Jindal AK, et al. Pulmonary presentation of Kawasaki disease-A diagnostic challenge. Pediatr Pulmonol 2018; 2018: 103-107

17. Toy P, Lowell C. TRALI - Definition, mechanisms, incidence and clinical relevance. Best Pract Res Clin Anaesthesiol 2007; 21: 183-193. 
18. Ibrahim J, Al Amri A, Ghatasheh G. Transfusion-related acute lung injury after immunoglobulin infusion for Kawasaki disease: a case report and literature review. Glob Pediatr Heal 2017; 4: $2333794 \times 17746545$.

19. Hetland LE, Susrud KS, Lindahl KH, et al. Henoch-schönlein purpura: A literature review. Acta Derm Venereol 2017; 97: 1160-1166.

20. Besbas N, Duzova A, Topaloglu R, et al. Pulmonary haemorrhage in a 6-year-old boy with Henoch-Schönlein purpura. Clin Rheumatol 2001; 20: 293-296.

21. Chaussain M, de Boissieu D, Kalifa G, et al. Impairment of lung diffusion capacity in Schönlein-Henoch purpura. J Pediatr 1992; 121: 12-16.

22. Jariwala MP, Laxer RM. Primary vasculitis in childhood: GPA and MPA in childhood. Front Pediatr 2018; 6: 1-11.

23. Filocamo G, Torreggiani S, Agostoni C, et al. Lung involvement in childhood onset granulomatosis with polyangiitis. Pediatr Rheumatol 2017; 15: 28.

24. Feragalli B, Mantini C, Sperandeo M, et al. The lung in systemic vasculitis: Radiological patterns and differential diagnosis. $\mathrm{Br}$ J Radiol 2016; 89: 20150992.

25. James KE, Xiao R, Merkel PA, et al. Clinical course and outcomes of childhood-onset granulomatosis with polyangiitis. Clin Exp Rheumatol 2017; 35: 202-208.

26. Fina A, Dubus JC, Tran A, et al. Eosinophilic granulomatosis with polyangiitis in children: Data from the French RespiRare ${ }^{\circledR}$ cohort. Pediatr Pulmonol 2018; 53: 1640-1650.
27. Cabral DA, Canter DL, Muscal E, et al. Comparing presenting clinical features in 48 children with microscopic polyangiitis to 183 children who have granulomatosis with polyangiitis (Wegener's): An ARChiVe Cohort Study. Arthritis Rheumatol 2016; 68: 2514-2526.

28. Camus P. The Drug-Induced Respiratory Disease Website: Pulmonary vasculitis or capillaritis. https://www.pneumotox. com/pattern/view/42/VI.d/pulmonary-vasculitis-orcapillaritis Date last accessed: 7 August 2020.

29. Koné-Paut I. Behçet's disease in children, an overview. Pediatr Rheumatol 2016; 14: 1-8.

30. Araujo DB, Borba EF, Silva CA, et al. Alveolar hemorrhage: Distinct features of juvenile and adult onset systemic lupus erythematosus. Lupus 2012; 21: 872-877.

31. Breda L, Nozzi M, Harari S, et al. Hypocomplementemic urticarial vasculitis (HUVS) with precocious emphysema responsive to azathioprine. J Clin Immunol 2013; 33: 891-895.

32. Haq SA, Pagnoux C. Infection-associated vasculitides. Int J Rheum Dis 2019; 22: 109-115.

33. Liu $Y$, Jesus AA, Marrero B, et al. Activated STING in a Vascular and Pulmonary Syndrome. N Engl J Med 2014; 371: 507-518.

34. Watkin LB, Jessen B, Wiszniewski W, et al. COPA mutations impair ER-Golgi transport and cause hereditary autoimmunemediated lung disease and arthritis. Nat Genet 2015; 47: 654-660 